\section{Orbital metastatic primary mediastinal neuroendocrine tumor: a histopathological case report}

\author{
Hind Alkatan, ${ }^{1}$ Ayman Ayoubi ${ }^{2}$ \\ 'Pathology and Laboratory Medicine \\ Department; ${ }^{2}$ Oculoplastic and Orbit \\ Division, King Khaled Eye specialist \\ Hospital, Riyadh, Saudi Arabia
}

\section{Abstract}

Neuroendocrine tumors most frequently involve the gastrointestinal tract and bronchopulmonary system. Few cases of presumed primary neuroendocrine tumors in the orbit have been reported so far and most of the orbital cases are actually metastatic.

We describe the unusual occurrence of this tumor in the orbit of a 16-year-old boy. The lesion was initially thought to be primary; however, the diagnosis of a metastatic orbital lesion was later supported by the histopathological appearance of his orbital biopsy, characteristic immunohistochemical profile and the presence of a primary mediastinal tumor. The patient did not have any symptoms suggestive of a carcinoid syndrome during the course of his disease. Unfortunately, tests showed lymph node involvement and distant metastatic lesions and he died from these a few months later while on palliative therapy.

\section{Introduction}

Carcinoid tumors are neoplasms that are believed to arise from neuroendocrine cells of the gastrointestinal mucosa and in other anatomical locations such as lungs, ovaries, thyroid and breasts. ${ }^{1}$ Neuroendocrine tumors (NET) are often referred to using the term carcinoid, but the newer WHO classification uses the term NET. The classification of lesions is based on size, localization, proliferation rate, differentiation and hormone production. Metastatic spread to the eye and ocular adnexae is relatively uncommon and primary orbital cases are also rare. ${ }^{2}$

We report a patient who presented with an orbital neuroendocrine tumor that was finally concluded to be metastatic. His systemic workup before his death revealed a primary chest lesion.

\section{Case Report}

A 16-year-old male presented with rapidly progressing right side painless proptosis and diplopia over the previous month. The patient was healthy prior to his presentation. There was no history of preceding trauma and no other symptoms such as fever, night sweats, loss of weight or appetite; there was no history of skin lesions.

Visual acuity was 20/30 (uncorrected) on the affected side. External examination showed $8 \mathrm{~mm}$ of proptosis on the right side using Hertel exophthalmometer at base 102 . There was limitation of right eye abduction, dilated conjunctival blood vessels temporally, mild superficial exposure keratopathy and optic disc swelling on the same side (Figure 1a). Initial systemic examination revealed cervical lympadenopathy on the right side. Lymph nodes were soft, rubbery and non-tender with no overlying skin changes. Initial clinical diagnosis was rhabdomyosarcoma in view of his age and the rapidly progressing tumor or lymphoma, also taking into consideration his lymphadenopathy and the painless proptosis.

Magnetic resonance imaging of the orbits showed a large right temporally located extraconal orbital mass $(3 \times 2.5 \mathrm{~cm})$ abutting the lateral rectus muscle and extending to the orbital apex. The mass was isodense to the adjacent muscles with faint marginal contrast enhancement (Figure 1b). The radiology report suggested the possibility of a neurogenic tumor or rhabdomyosarcoma. Orbital incisional biopsy through right lateral orbitotomy showed tumor lobules separated by fibrous stroma in the hematoxylin and eosin stained histopathology slides. The lobules consist of poorly differentiated cells with small round to cuboidal nuclei and abundant cytoplasm (Figure 2a). Mitotic figures were frequent (Figure 2b). The tumor cells expressed cytokeratin CK8/18, chromogranin (Figure 3a) and synaptophysin (Figure 3 b). Negative markers included desmin, myogenin, CD99, S-100, smooth muscle actin (SMA) and epithelial membrane antigen (EMA). Proliferation rate was $20 \%$ by Ki67. The final diagnosis was neuroendocrine tumor with histological grading grade 2 . The patient was referred to a general tertiary care center for tests and management. His further systemic workup did not reveal any gastrointestinal primary tumor, but a computerized tomography (CT) scan of his chest showed a heterogenous lobulated mediastinal mass measuring $11.6 \times 9 \times 6 \mathrm{~cm}$ compressing the bronchial branches in addition to multiple nodules involving the lung parenchyma bilaterally. His total body CT scan also showed metastatic bone lesions involving lumbar vertebrae, left side of the sacrum and right iliac bone. Bilateral multiple enlarged supraclavicular
Correspondence: Hind Alkatan, King Khaled Eye Specialist Hospital, Riyadh, Saudi Arabia. Tel: +966.1.4821234 Ext 3131; Mobile: +966504492399. E-mail: hkatan@kkesh.med.sa

Key words: neuroendocrine, carcinoid, orbit.

Acknowledgements: this paper was presented at the Eastern Ophthalmic Pathology Society (EOPS) Meeting, Cleveland, Ohio, USA, September 2010. IRB/EC: this case study was not registered with the IRB but followed the guidelines stipulated by the ethics committee of the institution.

Contributions: HA is fully responsible for the accuracy of the information and for preparation of the manuscript; AA has provided clinical data and is the treating physician responsible for the case.

Conflict of interests: the authors declare no potential conflict of interests.

Received for publication: 31 May 2011.

Revision received: 6 February 2012

Accepted for publication: 13 February 2012.

This work is licensed under a Creative Commons Attribution NonCommercial 3.0 License (CC BYNC 3.0).

(C) Copyright H. Alkatan and A. Ayoubi, 2012 Licensee PAGEPress, Italy

Eye Reports 2012; 2:e3

doi:10.4081/eye.2012.e3

lymph nodes were also evident by magnetic resonance imaging in addition to pararenal soft tissue lesions adjacent to the left kidney and dorsolateral to the right iliopsoas muscle.

The patient was considered to have stage 4 disease because of his distant metastasis with the primary lesion being in the chest. No tissue diagnosis was considered. Because of the wide-spread disease, exenteration was not performed and he was started on chemotherapy consisting of cisplatin and etoposide. Response to the $1^{\text {st }}$ cycle of treatment was poor, as documented in his medical records. He also received palliative radiotherapy to the supraclavicular lymph nodes and his enlarging right orbital lesion. He was given a total dose of 20$30 \mathrm{~Gy}$ in 5-10 fractions. He was then transferred to another local health care facility in his family's home town for supportive care. His condition deteriorated over the next few months and he died from his metastatic disease.

\section{Discussion}

NET originates from the chromaffin cells of 
the gastrointestinal and bronchial tract. ${ }^{1,3}$ Carcinoid syndrome may develop in a small number of patients, usually in association with metastatic disease, and it manifests as cutaneous flushing, cyanosis, diarrhea, asthma and endocardial fibrosis. ${ }^{4}$ Ophthalmic manifestations of this syndrome include conjunctival injection, intravascular sludging, perivascular pigment clumping and a decrease in the ophthalmic artery pressure that are believed to be attributed to serotonin and vasoactive peptides secreted by a functioning NET. ${ }^{5}$

Several cases of non-ocular NET in various anatomic locations have been reported; however, primary orbital NET is rare. ${ }^{1,2}$ Orbital involvement by NET accounts for only 4-5\% of all orbital metastatic disease and it is usually from a gastrointestinal primary tumor, while ocular uveal involvement is often from primary bronchopulmonary NETs. ${ }^{2,6}$ Bilateral orbital metastasis has been also reported. ${ }^{7,8}$

An overall high proportion of NET cases (15\%) metastasize to the orbit, although it is considered a relatively rare site for metastatic disease. ${ }^{9}$ Of these cases of orbital involvement by NET, the majority of the metastases are from primary gastrointestinal tumors or tumors of unknown primary origin. Mititelu et al. reported a presumed primary left orbital NET progressing to neoplastic meningitis while the original tumor site was never identified. ${ }^{10}$ Riddle et al. reported 15 cases of eye and
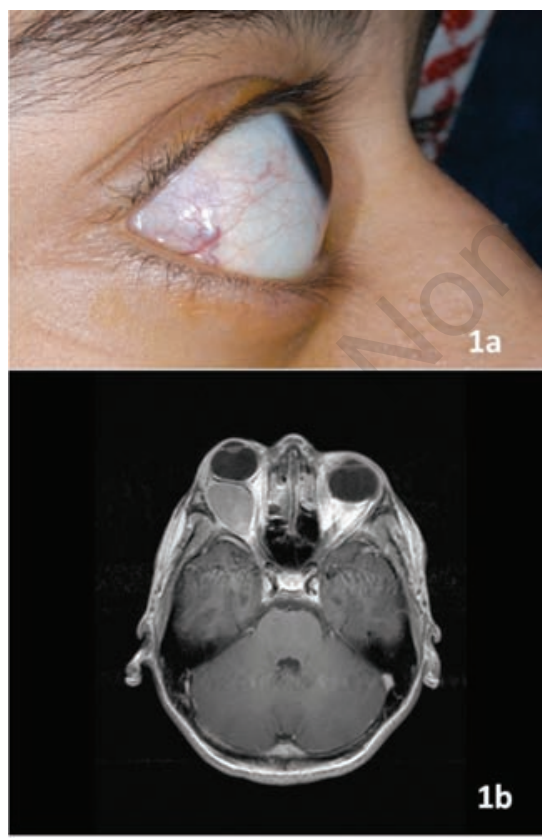

Figure 1. (a) Clinical appearance of the proptotic right globe in our 16-year-old patient. (b) Axial T1-magnetic resonance imaging showing the right isodense mass pushing the optic nerve, with ring of contrast enhancement. orbit NET metastases. Of the 12 cases in whom the primary site was known: 7 cases were bronchial, 4 from the ileum and one case only was from trachea. However, if we isolate these 7 orbital cases, the primary site was unknown in 3 , was ileum in 3 and trachea in one case only. ${ }^{11}$ In a review by Shetlar et al. of 18 cases of orbital metastases, including 3 cases reported by the authors and an additional 15 cases reported by others, 11 were of gastrointestinal origin, the primary site was unknown in 5 , mediastinal in one and trachea in one. ${ }^{4}$

Mehta et al. reported their 13 cases of orbital metastatic NET among whom 11 were of gastrointestinal origin (ileal in 7 and colonic in 4), and only one each from breast and bronchus. Mehta et al. also reviewed the literature of NET metastasis to the orbit and out of 23 cases reported by others (in which the primary tumor was known), 16 were of gastrointestinal origin, 5 of bronchopulmonary origin, one was of renal origin and one was of mediastinal origin. ${ }^{12}$

On the other hand, only a few cases of metastatic ocular uveal NET have been reported. ${ }^{11}$ Metastatic spread to the eye itself is more likely to arise from bronchial tumors, and it involves primarily the uveal tract where it can be misdiagnosed as uveal melanoma. ${ }^{4,13}$ The primary tumor in almost all uveal metastasis has been shown to arise from the bronchus. The reasons behind this predilection are not understood. Riddle et al. reported that out of 8 cases of metastasis to the ocular uveal tissue from NET, 7 were of bronchial origin and the remaining single case was of ileal origin. ${ }^{11}$ Out of 12 uveal metastatic NET reviewed by Shetlar et al., in contrast to the orbital cases in the same review discussed above, the primary site was bronchial in 11 cases and the ileum in one case only. ${ }^{4}$ In their review, Mehta et al. commented that this might be due to intrinsic properties of the tumors affecting their ability to develop a metastasis. ${ }^{12}$

Metastases are uncommon in typical bronchopulmonary NET, but regional lymph node secondary tumors occur more commonly in atypical settings; although distant metastasis occurs less frequently. Usual metastases are to lymph nodes, contralateral lung, liver, bone, ovaries, brain and skin. ${ }^{14}$ Metastatic intraocular or orbital NET usually presents after the primary neoplasm is diagnosed., ${ }^{2,11}$

Symptoms of the carcinoid syndrome are commonly observed in cases of metastatic disease in general, as functioning NET metastases (usually hepatic) may secrete vasoactive substances, detected as raised urinary 5-HIAA, and manifest clinically as symptoms of carcinoid syndrome, such as facial flushing, diarrhea, abdominal cramps, asthma and tachycardia. ${ }^{11,12}$ However, Carcinoid syndrome is rare in bronchpulmonary NET without metastasis; therefore, symptoms are usually absent. ${ }^{15}$

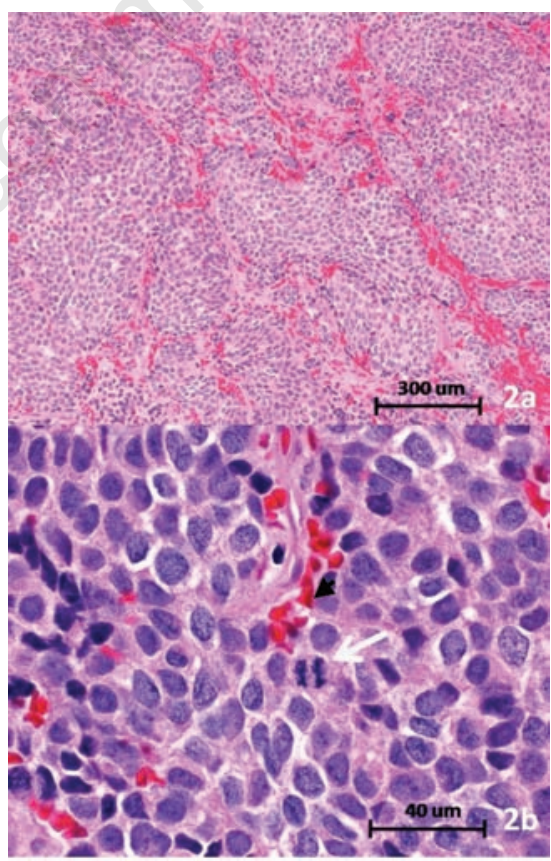

Figure 2. (a) Histopathology picture of the tumor lobules (Hematoxylin and Eosin, original magnification x 200). (b) Mitotic figure (white arrow) (Hematoxylin and Eosin, original magnification $x$ 1000).

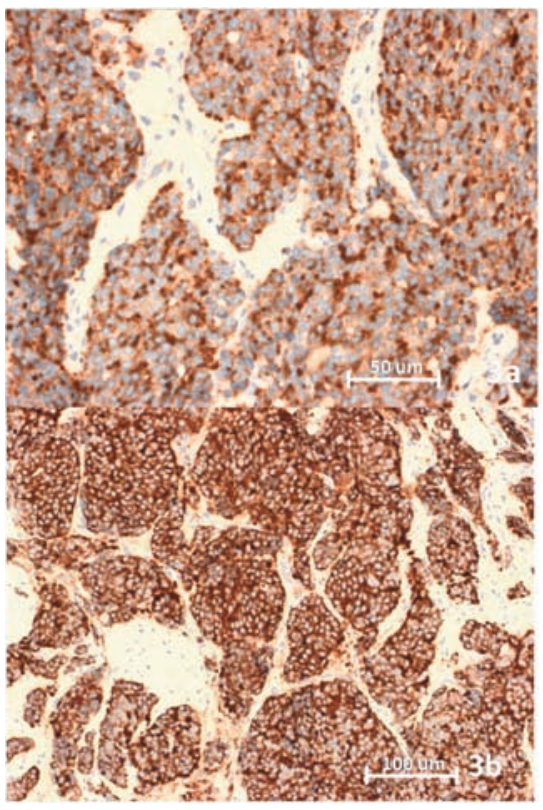

Figure 3. (a) Positivity of the tumor cells as indicated by dark brown staining to chromogranin A (original magnification $x$ 400). (b) Positive islands of tumor cells to synaptophysin stained brown within light colored stroma (original magnification $x$ 200). 
Zimmerman and his co-authors reviewed 15 cases of orbital/ocular metastatic NET and only 4 of these patients had elevated 5-HIAA and/or symptoms of the carcinoid syndrome. ${ }^{2}$ Even with metastasis to the orbit, symptoms of carcinoid syndrome are often absent. Only 2 patients out of the 13 cases of orbital metastatic NET reported by Mehta et al. had elevated urinary 5-HIAA without hepatic metastasis, thus suggesting a significant production of the vasoactive substances by the orbital metastatic tumor itself. ${ }^{12}$

The location of his ocular metastatic lesion makes our patient unique. His primary mediastinal NET was asymptomatic and he presented first with the orbital metastatic lesion before the primary lesion was discovered. This is considered unusual since the metastatic disease was orbital instead of being intraocular (uveal). Sira and co-authors similarly reported the first case of NET metastasizing from the larynx to the orbit; however, in their case the involvement was bilateral. ${ }^{8}$ Our patient also did not have symptoms suggestive of a carcinoid syndrome to attract attention to his primary disease. The absence of carcinoid syndrome manifestations delayed his diagnosis.

Another interesting but rare finding in metastatic NET is their presentation as orbital inflammation that must be carefully distinguished from the systemic carcinoid syndrome. This presentation is thought to be related to spontaneous release of intrinsic inflammatory mediators. ${ }^{8,16}$

The histological differential diagnosis suggested by Zimmerman et al. includes amelanotic melanoma, paraganglioma and metastasis from oat cell carcinoma, retinoblastoma, neuroblastoma, since they all share the histological cellular appearance of small dark cells, but can otherwise be eliminated according to clinical, immunohistochemical and ultrastructural criteria. The differential diagnosis also includes non-orbital NET; this is more problematic. ${ }^{2}$ Therefore, immunohistochemical methods are important for proper diagnosis with frequent reactivity to chromogranin A and synaptophysin. Chromogranin A is one of the acidic proteins present in the secretory granules of neuroendocrine cells and is seen in most NET, while synaptophysin is another broad-spectrum neuroendocrine marker localized in cytoplasmic vesicles in neurons, neuroendocrine cells and their neoplasms. Other useful markers for NET include neuron-specific enolase that is a highly sensitive, but not specific, marker for NET, in addition to serotonin and calcitonin for metastatic NET, depending on the characteristic hormone or marker related to the primary site. It has been suggested to use a selective panel of antibodies to obtain the greatest diagnostic accuracy. ${ }^{4}$ The differential diagnosis in our case, based on the histopathological appearance of the tumor and the clinical suspicion, included desmoplastic round cell tumor. This was ruled out by the lack of prominent desmoplastic stroma and muscular differentiation (negative desmin, SMA and myogenin). Our patient had positive staining to chromogranin A (polyclonal rabbit anti-human) and synaptophysin (monoclonal mouse antibody).

Magnetic resonance imaging is helpful in localizing the tumor which may demonstrate the same density as the extraocular muscles in both T1- and T2-weighted images. ${ }^{3}$ This was also seen in our case.

No exact data is available for long-term survival of ocular metastatic NET. The overall median survival of 227 cases of ocular metastatic disease reported by Ferry and Font was 7.4 months and only 2 out of these were NET. ${ }^{17}$ In general, a relatively good prospect for longterm survival in NET cases is expected, thus excision of small solitary metastatic lesions in the orbit is recommended to achieve local control of the disease. Enucleation or exenteration might also be ultimately required to ensure total surgical excision and local control of the disease that is believed to prolong survival time. ${ }^{3,4}$ The role of chemotherapy and external beam irradiation for orbital NET has not been well defined. However, some have reported an effective response to chemotherapy with cisplatin and etoposide. ${ }^{3}$

Our patient had incisional biopsy aimed at reaching the proper diagnosis before definitive surgical treatment was planned. However, he was found to have a late stage disease and only palliative therapy was offered to him in the referral hospital.

In conclusion, we report an unexpected metastatic NET in a patient with absent carcinoid syndrome manifestations. These factors helped a proper diagnosis to be made but also led to an unfavorable outcome. Periodic ophthalmological examination of patients with NET is recommended for early detection of any ocular involvement and to guide treatment. On the other hand, ophthalmologists should also be aware of the rare occurrence of these tumors in the orbit and eye in order to avoid any delay in management.

\section{References}

1. Godwin JD II. Carcinoid tumors: an analysis of 2837 cases. Cancer 1975;36:560-9.

2. Zimmerman LE, Stangl R, Riddle PJ. Primary carcinoid tumor of the orbit: a clinicopathologic study with histochemical and electron microscopic observations. Arch 0phthalmol 1983;101:1395-8.
3. Fan JT, Buettner H, Bartley GB, Bolling JP. Clinical features and treatment of seven patients with carcinoid tumor metastatic to the eye and orbit. Am J Opthalmol 1995; 119:211-8.

4. Shetlar DJ, Font RL, Ordonez N, et al. A clinicopathologic study of three carcinoid tumors metastatic to the orbit: Immunohistochemical, ultrastructural, and DNA flow cytometric studies. Ophthalmology 1990; 97:257-64.

5. Wong VG, Melmon KL. Ophthalmic manifestations of the carcinoid flush. N Engl J Med 1967;277:406-9.

6. Goldberg RA, Rootman J, Cline RA. Tumors metastatic to the orbit: a changing picture. Surv Ophthalmol 1990;35:1-24.

7. Sivagnanavel V, Riodan-Eva P, Jarosz J, et al. Bilateral orbital metastases from a neuroendocrine tumor. J Neuroophthalmol 2004;24:240-2.

8. Sira M, Clauss RP, Maclean C, Rose GE. Orbital metastases from neuroendocrine carcinoma, masquerading as graves orbitopathy. Orbit 2010;29:94-6.

9. Isidori AM, Kaltsas G, Frajese V, et al. Ocular metastases secondary to carcinoid tumors: the utility of imaging with [(123)I]meta-iodobenzylguanidine and [(111)In]DTPA pentetreotide. J Clin Endocrinol Metab 2002;87:1627-33.

10. Mititelu M, Stanton CA, Yeatts RP. Primary neuroendocrine tumor of the orbit progressing to neoplastic meningitis. Ophthal Plast Reconstr Surg 2008;24:231-3.

11. Riddle PJ, Font RI, Zimmerman LE. Carcinoid tumors of the eye and orbit: a clinicopathologic study of 15 cases, with histochemical and electron microscopic observations. Hum Pathol 1982;13:459-69.

12. Mehta JS, Abou-Rayyah Y, Rose GE. Orbital carcinoid metastasis. Ophthalmology 2006;113:466-72

13. Bardenstein DS, Char DH, Jones C, et al. Metastatic ciliary body carcinoid tumor. Arch Ophthalmol 1990;108:1590-4.

14. Aburn NS, Whitehead K, Sullivan TJ. Bronchpulmonary atypical carcinoid tumor metastatic to the orbit. Aust $\mathrm{N} Z \mathrm{~J}$ Ophthal mol 1995;23:241-4.

15. Conley YD, Cafoncelli AR, Khan JH, et al. Bronchial carcinoid tumor, experience over 20 years. Am J Surg 1992;58:670-2.

16. Knox RJ, Gigantelli JW, Arthurs BP. Recurrent orbital inflammation from metastatic orbital carcinoid tumor. 0phthalmic Plast Reconstr Surg 2001;17: 1379.

17. Ferry AP, Font R. Carcinoma metastatic to the eye and orbit. I. A clinicopathologic study of 227 cases. Arch Ophthalmol 1974;92:276-86. 